\title{
EFEITO DA ADIÇÃO DE CLORETO DE CÁLCIO SOBRE A QUALIDADE ESPERMÁTICA E ATIVIDADE DA ASPARTATO AMINO TRANSFERASE NO SÊMEN RESFRIADO DE SUÍNO ${ }^{1}$
}

\author{
Effect of adding calcium chloride on the spermatic quality and aminotransferase \\ aspartate in cool swine semen ${ }^{1}$
}

\author{
Fernanda Pinheiro Lima², Luis David Solis Murgas ${ }^{3}$, Silvio Luiz de Oliveira ${ }^{4}$, \\ Daniele de Lima ${ }^{5}$, Ana Luísa Neves Alvarenga ${ }^{6}$, Elias Tadeu Fialho ${ }^{7}$
}

\begin{abstract}
RESUMO
O experimento foi realizado com o objetivo de testar dois processos de resfriamento de sêmen suíno, analisar o efeito da adição de $\mathrm{CaCl}_{2}$ ao diluidor BTS e testar o método de avaliação do perfil enzimático da Aspartato Aminotransferase (AAT) sobre a qualidade espermática. Foram utilizados 12 ejaculados suínos de animais procedentes do setor de Suinocultura - DZO/UFLA. Estes ejaculados foram diluídos e receberam diferentes concentrações de $\mathrm{CaCl}_{2}$ (A: 0,0; B: 2,5; C: 5,0 e D: 7,5 mM). As amostras dos ejaculados foram submetidas a três processos de resfriamento ( 1 : convencional $-15^{\circ} \mathrm{C} ; 2$ : lento $-15^{\circ} \mathrm{C} / 5^{\circ} \mathrm{C} ; 3$ : rápido $-5^{\circ} \mathrm{C}$ ), sendo que cada ejaculado ficou armazenado por um período de 72 horas para avaliações da qualidade espermática, constituindo os tratamentos experimentais. Os parâmetros seminais avaliados foram motilidade e vigor espermáticos e perfil enzimático da AAT. Houve diferença significativa $(\mathrm{P}<0,05)$ quanto aos níveis de $\mathrm{CaCl}_{2}$ para o parâmetro de motilidade espermática, sendo que níveis maiores deste sal apresentaram resultados mais favoráveis à motilidade espermática. Para as variáveis vigor espermático e para o perfil da AAT não foi observado efeito da adição deste sal. Quanto ao processo de resfriamento, foi observada diferença significativa $(\mathrm{P}<0,05)$ para os parâmetros de motilidade e vigor espermáticos. Para o perfil enzimático não foi observado diferença significativa $(\mathrm{P}>0,05)$. Concluise que a adição de $\mathrm{CaCl}_{2}$ melhora a motilidade espermática das amostras dos ejaculados suínos e que o processo de resfriamento lento substitui o processo convencional sem afetar a qualidade espermática do sêmen submetido à refrigeração. A avaliação da AAT não é válida para sêmen resfriado.
\end{abstract}

Termos para indexação: Sêmen resfriado, suínos, parâmetros espermáticos.

\begin{abstract}
The study was carried out with objective to test two swine semen cooling processes and verify the effects of adding chloride of calcium $\left(\mathrm{CaCl}_{2}\right)$ on semen dilutor BTS and also to test the evaluation method of Aspartate Aminotransferase (AAT) enzymatic profile on the cooled swine semen spermatic quality. Were used twelve samples of ejaculation of breeders supplied by the Swine Breeding section at the DZO/UFLA. The samples were diluted and received different concentrations of $\mathrm{CaCl}_{2}(\mathrm{~A}: 0.0 ; \mathrm{B}: 2.5 ; \mathrm{C}$ 5.0; $\mathrm{D} 7.5 \mathrm{mM})$. The samples of ejaculation were submitted to three processes of cooling: 1 - standard cooling $\left(15^{\circ} \mathrm{C}\right) ; 2-$ slow cooling $\left(15^{\circ} \mathrm{C} / 5^{\circ} \mathrm{C}\right) ; 3$ - fast cooling $\left(5^{\circ} \mathrm{C}\right)$, and each sample of ejaculation was stored for a period of 72 hours to evaluation of spermatic quality, over all experimental treatments. The seminal parameters evaluated were the spermatic motility and strength and enzymatic profile of the AAT. A meaningful difference was verified $(\mathrm{P}<0.05)$ in the levels of $\mathrm{CaCl}_{2}$ for the parameters of spermatic motility, high levels of this salt showed better results in this parameter. The variables spermatic strength and AAT profile, shown no effects for any addition of $\mathrm{CaCl}_{2}$. For the cooling process, a meaningful difference was verified $(\mathrm{P}<0.05)$ in the parameters of spermatic motility and strength. No effects were observed concerning the enzymatic profile of the AAT ( $P>0.05)$. It was concluded that the adding of $\mathrm{CaCl}_{2}$ did improve the spermatic motility of the samples of swine ejaculations and that the process of slow cooling is able to replace the standard process without affecting the spermatic quality of semen submitted to cooling. The assessment of AAT is not valid to cooled semen.
\end{abstract}

Index terms: Cooled semen, swine, seminal parameters.

(Recebido em 4 de abril de 2006 e aprovado em 21 de novembro de 2006)

${ }^{1}$ Parte da dissertação de mestrado em Ciências Veterinárias do primeiro autor.

Médica Veterinária mestrado em Ciências Veterinárias - Departamento de Medicina Veterinária/DMV da Universidade Federal de Lavras/UFLA Cx. P. 3037 - 37200-000 - Lavras, MG - pinheiro_fl@yahoo.com.br

${ }^{3}$ Médico Veterinário, MSc, DSc, professor adjunto do Departamento de Medicina Veterinária/DMV da Universidade Federal de Lavras/UFLA - Cx. P. 3037 37200-000 - Lavras, MG - Ismurgas@ufla.br

${ }^{4}$ Médico Veterinário, MSc, DSc, professor da Universidade Federal de Goiás/UFG - Campus Jataí - 74001-970 - Jataí, GO - s3oliveirac@yahoo.com.br ${ }^{5}$ Acadêmica do curso de Medicina Veterinária, Departamento de Medicina Veterinária/DMV da Universidade Federal de Lavras/UFLA - Cx. P. 3037 37200-000 - Lavras, MG - danivet2003@yahoo.com.br

${ }^{6}$ Médica Veterinária, mestranda em Ciências Veterinárias do Departamento de Medicina Veterinária/DMV da Universidade Federal de Lavras/UFLA - Cx. P. 3037 -37200-000 - Lavras, MG - analuisaneves@hotmail.com

${ }^{7}$ Engenheiro Agrônomo, PhD, professor titular do Departamento de Zootecnia/DZO da Universidade Federal de Lavras/UFLA - Cx. P. 3037 - 37200-000 Lavras/MG - fialho@ufla.br 


\section{INTRODUÇÃO}

A dificuldade de preservação de sêmen suíno por períodos prolongados, sem alterar sua capacidade fecundante, limita a expansão da técnica de Inseminação Artificial (IA), visto que a tecnologia de criopreservação do sêmen suíno não está ainda tão bem consolidada quanto à do sêmen bovino. Em suínos, a maior parte das práticas de IA faz-se utilizando sêmen resfriado, mantido a uma temperatura entre 15 a $18^{\circ} \mathrm{C}$ até o terceiro dia após a colheita e a diluição. Devido às dificuldades decorrentes da manutenção da temperatura de armazenamento, novas metodologias têm sido estudadas para viabilizar o uso de sêmen resfriado a $5^{\circ} \mathrm{C}$, ou para aperfeiçoar o processo de congelamento.

A tecnologia de colheita, avaliação, manipulação e conservação do sêmen adotada nos programas de IA segue certo padrão mundial. A colheita é realizada pelo método da mão enluvada (KING \& MACPHERSON, 1973), com auxílio de manequim; a avaliação da qualidade do sêmen através de parâmetros para volume do ejaculado, motilidade, vigor, concentração e morfologia espermáticos; doses inseminantes de 2,5 a 3,5 bilhões de espermatozóides e com um volume entre 80 a $100 \mathrm{~mL}$; conservação na temperatura de $15-18^{\circ} \mathrm{C}$ e preservação pelo período médio de três dias.

Em termos gerais, as exigências mínimas para um reprodutor doador de sêmen incluem motilidade espermática mínima de $70 \%$, vigor três, alterações morfológicas com menos de $20 \%$ de formas anormais, volume e número total de espermatozóides do ejaculado mínimo de $100 \mathrm{~mL}$ e 3060 bilhões, respectivamente, dependendo da idade e do ritmo de colheita, e capacidade de conservação com mais de $60 \%$ de motilidade espermática após 48 horas de armazenamento. Segundo Silveira \& Scheid (2003), na prática uma taxa de oito a $23 \%$ dos reprodutores suínos não preenchem esses critérios.

O cálcio é o metal iônico mais abundante e um dos mais importantes elementos constitucionais do organismo e da fisiologia celular, sendo que suas funções podem ser divididas em extra e intracelular, dependendo de seu sítio de ação (GILMAN et al., 1991). O cálcio intracelular, no espermatozóide, tem um papel fundamental nos processos de capacitação, hiperatividade e reação acrossomal para a fertilização. Além disso, o cálcio intracelular é o principal elemento para a motilidade flagelar e fusão da vesícula acrossomal (DARSZON et al., 1999).

A motilidade hiperativada é definida como o padrão de movimento flagelar apresentado pelo espermatozóide no sítio de fertilização. O cálcio possui uma importante função na regulação da motilidade hiperativada (SUAREZ \& HO, 2003), atuando principalmente no axonema, mas pouco se sabe de seu modo de ação. A membrana plasmática apresenta canais de cálcio voltagemdependentes que iniciam e mantêm o aumento intracelular deste íon na hiperativação. O movimento flagelar necessita também do aumento de trifosfato de adenosina (ATP) e adenosina monofosfato cíclico (AMPc) (BOOTMAN et al., 2001).

Os métodos tradicionalmente utilizados para predizer a capacidade fecundante dos espermatozóides de um ejaculado apresentam resultados muitas vezes insatisfatórios, quando avaliados individualmente. A motilidade e a fertilidade são as técnicas mais utilizadas para avaliar o potencial reprodutivo dos reprodutores. Porém, o resultado da fertilidade do animal requer um considerável número de animais, tempo e recursos financeiros. Além disso, as diferenças peculiares entre as fêmeas podem confundir a interpretação dos resultados (BORQUE \& AYLLÓN, 1996).

A função metabólica da AAT está relacionada à participação da enzima nos processos energéticos dos espermatozóides. Nas células prostáticas, a enzima participa da produção de citrato (COSTELLO et al., 1988; citados por CIERESZKO et al., 1990).

Desta forma, a análise laboratorial da AAT pode ser um dos critérios utilizados para avaliar a capacidade fertilizante dos espermatozóides, uma vez que esta enzima apresenta fundamental importância para o seu metabolismo, relacionado com a região intermediária da cauda da célula espermática, denominada de bainha mitocondrial (MORENO et al., 2001).

Realizou-se este trabalho objetivando testar novos processos de resfriamento de sêmen suíno, analisar o efeito da adição de cloreto de cálcio sobre os parâmetros espermáticos e validar o teste de perfil enzimático da enzima AAT para caracterizar a qualidade espermática de ejaculados submetidos ao processamento e manipulação.

\section{MATERIAL E MÉTODOS}

Foram realizadas 12 colheitas de quatro reprodutores suínos mestiços das raças Large-White X Landrace, com idade média entre um e dois anos. Os reprodutores utilizados neste trabalho encontravam-se em serviço regular (um ejaculado por semana em média) no setor de Suinocultura do DZO/UFLA. Após a colheita, o volume do ejaculado foi medido diretamente pela graduação do copo coletor. $\mathrm{O}$ sêmen foi colocado em banho-maria a uma temperatura de $37^{\circ} \mathrm{C}$ por um período de 20 minutos. Foram realizadas determinação da concentração espermática utilizando a 
câmara de Newbauer e cálculo das doses inseminantes, para em seguida se proceder a diluição do ejaculado com diluidor de sêmen BTS.

O sêmen foi então fracionado em quatro provetas pré-aquecidas para o recebimento dos níveis de cloreto de cálcio: $\mathrm{A}$ - controle $\left(\mathrm{sem} \mathrm{CaCl}_{2}\right) ; \mathrm{B}-\mathrm{CaCl}_{2} 2,5 \mathrm{mM} ; \mathrm{C}-$ $\mathrm{CaCl}_{2}$ 5,0 mM; D- $\mathrm{CaCl}_{2}$ 7,5 mM. Nesta fase então o sêmen foi envasado em bisnagas contendo $80 \mathrm{~mL}$ cada uma, permanecendo por duas horas em local escuro à temperatura ambiente $\left(25^{\circ} \mathrm{C}\right)$. A dose inseminante foi fixada em uma concentração de cinco bilhões de espermatozóides.

Após este período de repouso, uma parcela dos preparados foi incubada em geladeira a $15^{\circ} \mathrm{C}$ (refrigeração convencional), de onde foram retiradas amostras no tempo em intervalos pré-determinados para a realização das avaliações. A outra parcela dos preparados foi levada diretamente à geladeira a $5^{\circ} \mathrm{C}$ (refrigeração rápida), e foram realizadas avaliações nos mesmos intervalos propostos para a refrigeração convencional, sendo que uma parcela das amostras que ficaram a $15^{\circ} \mathrm{C}$, também foram retiradas após permanecerem 24 horas nesta temperatura e levadas à geladeira de $5^{\circ} \mathrm{C}$ (refrigeração lenta), de onde foram retiradas amostras nos mesmos intervalos de tempo.

A motilidade geral e o vigor foram avaliados, utilizando escalas de 0 a 100 e 0 a 5 respectivamente, segundo metodologia proposta por Scheid (1993). Sendo as leituras realizadas em microscópio óptico (40x). As variáveis motilidade e vigor espermáticos foram analisados até 72 horas após a colheita. Todas as avaliações foram realizadas em triplicata, independemente, por dois avaliadores e expressas em percentual de células móveis na amostra, sendo que as amostras eram préaquecidas antes de cada avaliação. As coletas de amostras de ejaculados para determinação do perfil enzimático da AAT foram feitas até um período de 48 horas após a colheita de sêmen.

Para avaliação do perfil enzimático foi utilizado o Kit Doles Bioquímica Clínica ${ }^{\circledR}$, segundo a metodologia proposta por Reitman \& Frankel (1957).

Para as variáveis motilidade e vigor espermáticos foram adotados o teste de qui-quadrado para verificar a freqüência dos dados já que estes dados não foram significativos ao teste de normalidade e nem passíveis de transformação. Para o perfil enzimático, foi aplicado o teste F, uma vez que não houve diferença entre as médias observadas. As análises estatísticas foram realizadas utilizando-se o programa SAS Institute (1995).

\section{RESULTADOS E DISCUSSÃO}

Os valores médios da motilidade e vigor espermáticos, das amostras que receberam os diferentes níveis de cloreto de cálcio, estão representados na Tabela 1. As amostras de ejaculados que receberam os maiores níveis de $\mathrm{CaCl}_{2}(5,0$ e $7,5 \mathrm{mM})$ tiveram maiores freqüências $(77,08$ e 77,78\%, respectivamente) com valores de motilidade espermática significativamente maiores $(\mathrm{P}<0,05)$ que os demais níveis, pelo teste de qui-quadrado. Porém, com relação ao vigor espermático não foi observado significância $(\mathrm{P}>0,05)$ entre os níveis de $\mathrm{CaCl}_{2}$.

Toniolli \& Combarnous (1999), trabalhando com tecnologia de sêmen, estudaram também o efeito da adição de cloreto de cálcio ao sêmen suíno. Esses autores utilizaram níveis de $\mathrm{CaCl}_{2}$ diferentes $(0 ; 0,041$; 0,$41 ; 4,1$ e $41 \mathrm{mM}$ ) e observaram que concentrações maiores de 4,1 e $41 \mathrm{mM}$ deste sal promoveram valores de motilidade maiores aos das outras concentrações até o terceiro dia de acondicionamento. Estes autores concluíram também que altas concentrações deste sal no meio extracelular têm um efeito negativo sobre a motilidade espermática. No presente experimento os níveis de 5,0 e 7,5 $\mathrm{mM}$ de $\mathrm{CaCl}_{2}$ influenciaram a motilidade espermática, mantendo maiores freqüências de motilidade na escala de $60-90 \%$.

O íon cálcio tem um papel regulador no controle da motilidade e do metabolismo energético do espermatozóide. Ele é responsável por modificações bioquímicas importantes para a função normal do espermatozóide (NISHIMURA, 1993). Após a ejaculação, os espermatozóides passam por um processo de capacitação e a reação acrossômica é acompanhada de um aumento da concentração intracelular de cálcio. No reprodutor suíno, as modificações da permeabilidade de membrana aos íons e, em particular, ao cálcio durante a capacitação, seriam responsáveis pela mudança da motilidade dos espermatozóides (TONIOLLI \& COMBARNOUS, 1999).

Os resultados de motilidade e vigor espermáticos obtidos durante o processo de resfriamento são apresentados na Tabela 2. Observou-se um efeito significativo $(\mathrm{P}<0,05)$ do tipo de refrigeração sobre as características físicas do sêmen, motilidade e vigor espermáticos, sendo que a utilização dos processos de refrigeração convencional $\left(15^{\circ} \mathrm{C}\right)$ e resfriamento lento $\left(15^{\circ}\right.$ $\left.\mathrm{C} / 5^{\circ}\right)$ proporcionaram as maiores freqüências $(83,85 \mathrm{e}$ $80,21 \%$, respectivamente) com valores de motilidade espermática maiores que o processo de refrigeração rápida $\left(5^{\circ} \mathrm{C}\right)(49,48 \%)$ na faixa de motilidade de $60-90 \%$. 
TABELA 1 - Valores médios para motilidade (\%) e vigor espermáticos (1 a 5) das amostras dos ejaculados que receberam diferentes níveis de $\mathrm{CaCl}_{2}$ até 72 horas após a coleta.

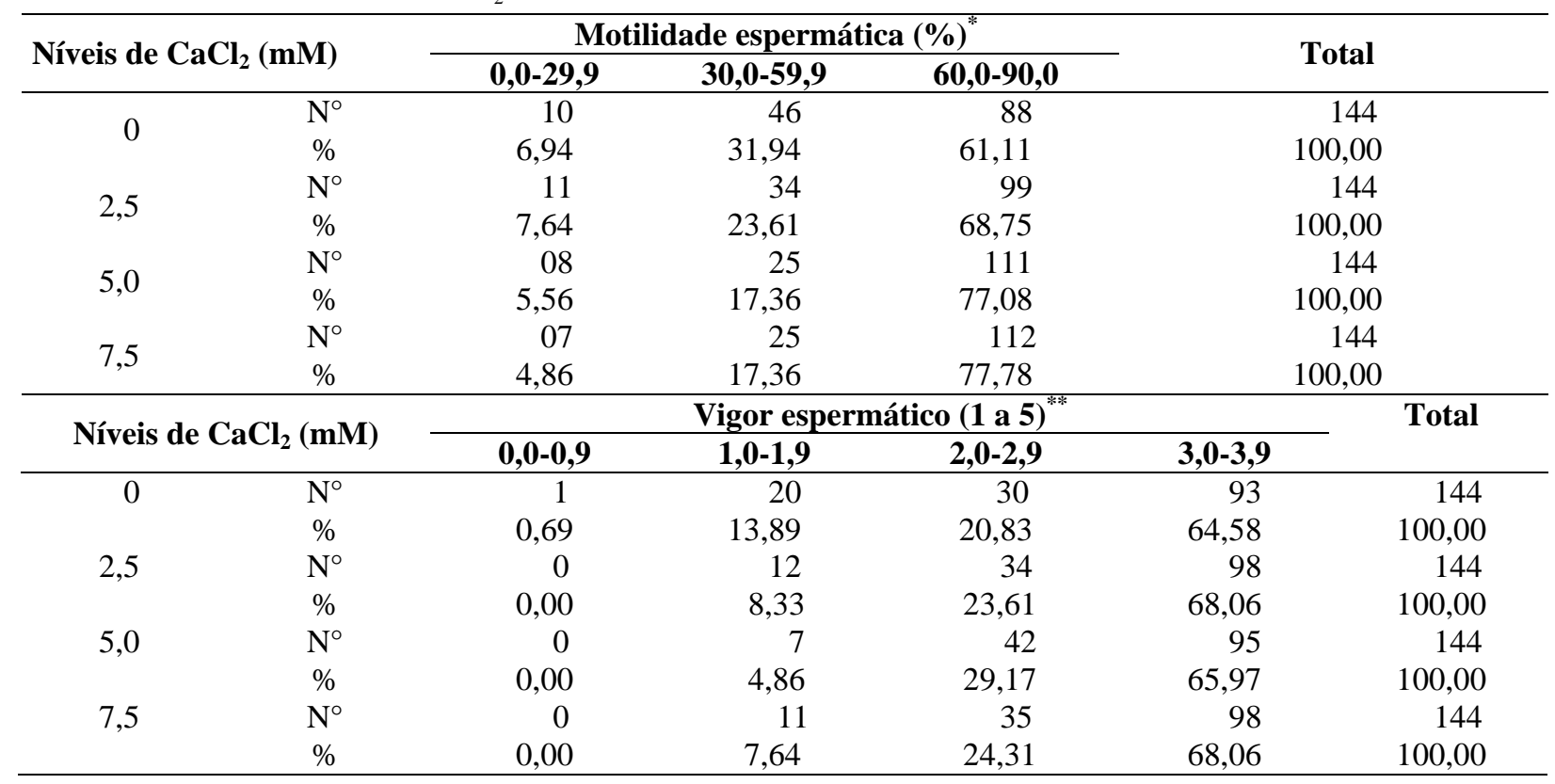

${ }^{*}$ Teste de Qui-quadrado $(\mathrm{P}<0,05),{ }^{* *}$ Teste de Qui-quadrado $(\mathrm{P}>0,05) . \mathrm{N}=$ análises realizadas.

TABELA 2 - Valores médios para motilidade (\%) e vigor espermáticos (1 a 5) das amostras dos ejaculados que passaram por diferentes processos de resfriamento até 72 horas após a coleta.

\begin{tabular}{|c|c|c|c|c|c|c|}
\hline \multirow{2}{*}{\multicolumn{2}{|c|}{ Tipo de refrigeração }} & \multicolumn{3}{|c|}{ Motilidade espermática (\%) } & \multirow{2}{*}{\multicolumn{2}{|c|}{ Total }} \\
\hline & & $\mathbf{0 , 0 - 2 9 , 9}$ & $30,0-59,9$ & $60,0-90,0$ & & \\
\hline \multirow[t]{2}{*}{$15^{\circ} \mathrm{C}$} & $\mathrm{N}^{\circ}$ & 7 & 24 & 161 & \multicolumn{2}{|c|}{192} \\
\hline & $\%$ & 3,65 & 12,50 & 83,85 & & \\
\hline \multirow[t]{2}{*}{$15^{\circ} \mathrm{C} / 5^{\circ} \mathrm{C}$} & $\mathrm{N}^{\circ}$ & 0 & 38 & 154 & \multicolumn{2}{|c|}{192} \\
\hline & $\%$ & 0,00 & 19,79 & 80,21 & \multicolumn{2}{|c|}{100,00} \\
\hline \multirow[t]{2}{*}{$5^{\circ} \mathrm{C}$} & $\mathrm{N}^{\circ}$ & 29 & 68 & 95 & \multirow{2}{*}{\multicolumn{2}{|c|}{$\begin{array}{r}192 \\
100,00\end{array}$}} \\
\hline & $\%$ & 15,10 & 35,42 & 49,48 & & \\
\hline \multicolumn{2}{|c|}{ Tipo de refrigeração } & \multirow{2}{*}{\multicolumn{3}{|c|}{\begin{tabular}{lr}
\multicolumn{2}{|c|}{ Vigor espermático (1 a 5) } \\
$1,0-1,9$ & $2,0-2,9$
\end{tabular}}} & & \multirow[t]{2}{*}{ Total } \\
\hline & & & & & $3,0-3,9$ & \\
\hline \multirow{2}{*}{$15^{\circ} \mathrm{C}$} & $\mathrm{N}^{\circ}$ & 0 & 17 & 37 & 138 & 192 \\
\hline & $\%$ & 0,00 & 8,85 & 19,27 & 71,88 & 100,00 \\
\hline \multirow[t]{2}{*}{$15^{\circ} \mathrm{C} / 5^{\circ} \mathrm{C}$} & $\mathrm{N}^{\circ}$ & 0 & 7 & 44 & 141 & 192 \\
\hline & $\%$ & 0,00 & 3,65 & 22,92 & 73,44 & 100,00 \\
\hline \multirow[t]{2}{*}{$5^{\circ} \mathrm{C}$} & $\mathrm{N}^{\circ}$ & 1 & 26 & 60 & 105 & 192 \\
\hline & $\%$ & 0,52 & 13,54 & 31,25 & 54,69 & 100,00 \\
\hline
\end{tabular}

*Teste de Qui-quadrado $(\mathrm{P}<0,05) \mathrm{N}^{\circ}=$ análises realizadas.

O vigor espermático comportou-se da mesma maneira, sendo que na escala de 3-4 os processos de refrigeração convencional e o resfriamento lento apresentaram, respectivamente, freqüências de 71,88 e $73,44 \%$, enquanto com a utilização do resfriamento rápido as freqüências obtidas, nesta escala, foram de apenas $54,69 \%$.

No presente trabalho foi observado que o processo de resfriamento rápido, utilizando o diluente BTS, não é 
tão eficiente para preservar as características seminais (motilidade e vigor espermáticos) quanto o resfriamento convencional e o lento. Segundo Sone et al. (1992), a utilização do diluidor BTS está condicionada ao processo de resfriamento convencional $\left(15-17^{\circ} \mathrm{C}\right)$, sendo que o terceiro dia após a colheita aparece como o limite máximo de utilização compatíveis com níveis ideais de fertilidade e de prolificidade para os criadores. Com o declínio da temperatura, há uma redução inevitável na proporção de espermatozóides que permanecem com integridade de membrana e componentes ultra-estruturais e bioquímicos normais (JOHNSON et al., 2000).

Corrêa et al. (2005) avaliaram o efeito de um novo diluente para testar duas temperaturas de acondicionamento do sêmen suíno $\left(5^{\circ}\right.$ e $\left.17^{\circ} \mathrm{C}\right)$. Os resultados mostraram que o diluente testado (PIGPEL-5) é capaz de manter a viabilidade espermática após o armazenamento a $5^{\circ} \mathrm{C}$, comparado com sêmen acondicionado em BTS a $17^{\circ} \mathrm{C}$, quando considerados a motilidade, o vigor e a morfologia espermáticos, teste de estresse térmico e o teste de choque osmótico. Estes autores também utilizaram a tabela de distribuição de frequiências para avaliação destas variáveis.

No presente trabalho, 49,48\% das amostras apresentaram motilidade espermática entre $60-90 \%$ no processo de resfriamento a $5^{\circ} \mathrm{C}$. Este resultado mostra que nesta temperatura ocorre uma redução da motilidade espermática, provavelmente, desencadeada pelo estresse do resfriamento rápido. Graham (1996), estudando o resfriamento do sêmen eqüino a $5^{\circ} \mathrm{C}$, afirmou que a motilidade espermática e, conseqüentemente, a capacidade fecundante pode ser prejudicada pelo estresse térmico que afeta a distribuição de componentes lipoprotéicos e a permeabilidade da membrana espermática.

Os resultados da análise de variância não mostraram efeito significativo $(\mathrm{P}>0,05)$ para o perfil de AAT no plasma seminal. Os valores encontrados para a concentração de AAT no presente trabalho são apresentados na Tabela 3. Neste estudo, o estresse causado pelo resfriamento não foi suficiente para promover diferenças nos níveis de AAT liberados.

Borque \& Ayllón (1996), trabalhando com sêmen de carneiros, submeteram as células espermáticas ao que foi chamado de estresse mínimo (centrifugação da amostra de sêmen fresco) e estresse máximo (congelamento e descongelamento sucessivos), e encontraram um efeito significativo $(\mathrm{P}<0,01)$ na atividade enzimática da AAT depois que os ejaculados foram submetidos ao processo de estresse máximo. No entanto, num estudo conduzido por Ciereszko et al. (1992), para avaliar e caracterizar a atividade da AAT no sêmen de reprodutor suíno e determinar o efeito na taxa de diluição foi encontrado que a atividade da AAT que tinha saído do espermatozóide aumentava gradualmente durante o período de estoque em vários diluentes a uma temperatura de $16-18^{\circ} \mathrm{C}$. Todos estes achados sugerem que para o sêmen suíno resfriado a validade da técnica de avaliação do perfil enzimático é controversa. Sugere-se que a análise da atividade enzimática da AAT seja melhor observada quando as células espermáticas são submetidas a um processo de estresse, causado pelo congelamento, ou pelo resfriamento em períodos mais prolongados do que os que foram utilizados no presente experimento.

TABELA 3 - Valores médios para o perfil enzimático da AAT (UI/ml) e erro padrão da média de acordo com os níveis de $\mathrm{CaCl}_{2}$ e com os tipos de resfriamento aos quais as amostras de ejaculados foram submetidas.

\begin{tabular}{cc}
\hline Níveis de $\mathbf{C a C l}_{\mathbf{2}} \mathbf{( m M )}$ & Média $^{*}$ \\
\hline 0 & $2,44(0,19)$ \\
2,5 & $2,75(0,19)$ \\
5,0 & $2,75(0,19)$ \\
7,5 & $2,56(0,19)$ \\
\hline Tipo de resfriamento & Média $^{*}$ \\
\hline $15^{\circ} \mathrm{C}$ & $2,55(0,17)$ \\
$15^{\circ} \mathrm{C} / 5^{\circ} \mathrm{C}$ & $2,70(0,17)$ \\
$5{ }^{\circ} \mathrm{C}$ & $2,64(0,17)$ \\
\hline
\end{tabular}

*Não houve diferença significativa entre as médias (teste F).

\section{CONCLUSÕES}

Nas condições em que este experimento foi realizado, pode-se concluir que o cloreto de cálcio só é capaz de preservar a motilidade espermática nas maiores concentrações deste sal (5,0 e 7,5 mM); a avaliação da AAT não é válida para sêmen resfriado suíno e que o processo de resfriamento lento substitui o processo convencional sem afetar a qualidade do sêmen submetido à refrigeração.

\section{REFERÊNCIAS BIBLIOGRÁFICAS}

BOOTMAN, M. P.; COOLLINS, T. J.; PEPPIATT, C. M.; PROTHERO, L. S.; MACKENZIE, L.; SMET, P.; TRAVERS, M.; TOVEYS, S. C.; SEO, J. T.; BERRIDGE, M. J.; CICCOLINI, F.; LIPP, P. Calcium signaling: an overview. Seminars in Cell and Developmental Biology, [S.1.], v. 12, p. 3-10, 2001. 
BORQUE, C.; AYLLÓN, A. Aspartate aminotransferase in ejaculates of manchego and merino rans after minimal and maximal sperm damage. Theriogenology, [S.1.], n. 46, p. 1017-1025, 1996.

CIERESZKO, A.; GLOGOWSKI, J.; STRZEZEK, J.; DEMIANOWICZ, W. Low stability of aspartate aminotransferase activity in boar semen. Theriogenology, [S.1.], n. 37, p. 1269-1281, 1992.

CIERESZKO, A.; JABLONOWSKA, C.; STRZEZEK, J. Aspartate aminotransferase activity in motile and immotile spermatozoa fractions of frozen-thawed boar semen obtained after filtration on columns filled with chitin. Animal Reproduction Science, Amsterdam, v. 23, n. 3, p. 237-244, Nov. 1990.

CORRÊA, M. N.; BIANCHI, I.; LUCIA JÚNIOR, T.; SERRET, C. G.; BORDIGNON, J.; RAMBO, G.; DESCHAMPS, J. C. Efeito do sêmen acondicionado a $5^{\circ} \mathrm{C}$ com o diluente PIGPEL-5 sobre a taxa de penetração espermática in vitro de ovócitos vitrificados. In: CONGRESSO BRASILEIRO DE VETERINÁRIOS ESPECIALISTAS EM SUÍNOS, 12., 2005, Fortaleza. Anais... Fortaleza: UFCE, 2005. p. 275-276.

DARSZON, A.; LABARCA, P.; ISHIGAKI, T.; ESPINOSA, F. Íon channels in sperm physiology. Physiological Review, [S.1.], v. 79, n. 2, p. 481-510, 1999.

GILMAN, A. G.; RALL, T. W.; NIES, A. S.; TAYLOR, P. As bases farmacológicas da terapêutica. 8. ed. Rio de Janeiro: Guanabara Koogan, 1991. 1232 p.

GRAHAM, J. K. Cryopreservation of stallion spermatozoa. Veterinary Clinic North American Equine Practice, [S.1.], v. 12, p. 131-147, 1996.

JOHNSON, L. A.; WEITZE, K. F.; FISER, P.; MAXWELL, W. M. C. Storage of boar semen. Animal Reproduction Science, [S.1.], v. 62, p. 143-172, 2000.

KING, G. I.; MACPHERSON, I. W. A comparison of two methods for boar semen collection. Journal of
Animal Science, Chamapign, v. 36, n. 4, p. 563-565, Apr. 1973.

MORENO, F. A. B.; BISPO, C. A. S.; GUERRA, M. M. P.; TENÓRIO FILHO, F.; OLIVEIRA, R. R.; ALVES, L. C.; WISCHRAL, A. Motilidade, vigor e dosagem de Aspartato Amino Transferase no sêmen caprino diluído em leite desnatado e Fiser. Revista Brasileira de Reprodução Animal, Belo Horizonte, v. 25, n. 3, p. 434435, 2001.

NISHIMURA, K. Effects of calcium íons on the malateaspartate shuttle in slow-cooled boar spermatozoa. Biology Reproduction, [S.1.], v. 49, p. 537-543, 1993.

REITMAN, S.; FRANKEL, S. A colorimetric method for the determination of serum glutamic oxalacetic and glutamic pyruvic transaminase. American Journal Clinical Pathology, [S.1.], p. 28-56, 1957.

SAS INSTITUTE. Guide for personal computers. Cary, 1985.

SCHEID, I. R. Manual de inseminação artificial de suínos: procedimentos e métodos de laboratório. Concórdia: CNPSA/Embrapa, 1993. 48 p.

SILVEIRA, P. R.; SCHEID, I. R. Qualidade de sêmen no processo de inseminação artificial. Suinocultura Industrial, [S.1.], n. 6, p. 33-38, 2003.

SONE, M.; CHIKYU, M.; YOSHIDA, M. Storage of boar semen in: liquid form. Japan Journal Swine Science, [S.1.], v. 29, p. 41-50, 1992.

SUAREZ, S. S.; HO, H. C. Hyperactivation of mammalian sperm. Cellular and Molecular Biology, [S.l.], v. 49, n. 3, p. 351-356, 2003.

TONIOLLI, R.; COMBARNOUS, Y. Adição de cálcio $\left(\mathrm{CaCl}_{2}\right)$ ao diluidor do sêmen do reprodutor: efeito sobre a motilidade espermática. Revista Brasileira de Reprodução Animal, Belo Horizonte, v. 23, n. 1, p. 33-40, jan./mar. 1999. 